\title{
GI-MS48-P08 | Olex2: Teaching New Software Old AND New Tricks
}

Puschmann, Horst (OlexSys Ltd, Durham, GBR); Dolomanov, Oleg (OlexSys Ltd, Durham, GBR)

Olex2 [1] is now firmly established as a major software package in the field of small-molecule crystallography: new and some (open-minded) old dogs find it a very useful and user-friendly platform from which to perform all sorts of tricks.

This contribution will be in two parts. In the first part, I will present some of the new tricks we have included in Olex2 over the last few years, including the streamlined management of solvent masking, fast refinement using our very own refinement engine olex2.refine [2] and the refinement of standard X-ray structures using nonspherical form factors.

In the second part, I will convince you that Olex2 is the perfect open-source and free platform that YOU can use to teach New Dogs Old Tricks: Programming Olex2 is quite easy and accessible to all, and if you've got an Old Trick, why not consider using Olex2 as a platform to implement it rapidly and then make these available to all of our users?

[1] OLEX2: a complete structure solution, refinement and analysis program OV Dolomanov, LJ Bourhis, RJ Gildea, JAK Howard, H Puschmann Journal of Applied Crystallography 42 (2), 339-341

[2] The anatomy of a comprehensive constrained, restrained refinement program for the modern computing environment-Olex2 dissected

LJ Bourhis, OV Dolomanov, RJ Gildea, JAK Howard, H Puschmann

Acta Crystallographica Section A: Foundations and Advances 71 (1), 59-7 